of genetic expression of clusters of related genes. He concluded that in E. coli and Salmonella typhimurium up to 70 per cent of the genes showed some clustering, but that in Pseudomonas aeruginosa it is quite rare. It was expensive, he thought, for each gene to have its own operator and so, without elustering, it was reasonable to oxpect regulation of enzyme synthesis at the translation level-a point arrived at by the previous speaker. W. H. Filliott also suggested control of ribonuclease synthesis in Bacillus subtilis at a stage later than transcription. He has found that actinomycin stimulates ribonuclease production-an observation which would seem to preclude control at the transcription stage. The presence in the cell of a firmly complexing inhibitor of the active ribonuclease secreted by this organism suggests that this enzyme is synthesized close to the cell membrane-from which it is released to the medium. Dr. Elliott also gave evidence for the synthesis of the exo-enzyme $\alpha$-amylase close to the cell membrane.

The regulation of RNA biosynthesis in general was reviewed by L. R. Finch, who added the idea that the present concepts of the utilization of $m \mathrm{RNA}$ for protein synthesis, before its own transcription from DNA was completed, allow possible alternative methods of repres sion not immodiately connected to the operator region. He saw combination of the repressor with the nascent peptide chain as offering more specificity than the simple operator region hypothesis.

G. L. Ada spoke of the structure of immune $\gamma$-globulin and the problems besetting the investigation of the mechanisms controlling the synthesis of antibody by cells from the spleen and lymph nodes. The heterogeneous nature of the lymph nodes makes conventional methods of following protein synthesis difficult to interpret in terms of antibody synthesis, but the use of radio-autography and specific fluorescent staining offers hope. Injection of powerful bacterial antigens labelled with radio-iodine into rats leads to the uptake of less than four molecules of antigen per plasma cell. It was inferred that intact antigen does not act as a template in the synthesis of antibody. The association of antigen with RNA as being involved as an intermediato in the antigenic response wrs discussed.

Attempts to define the number and function of proteins coded for by poliovirus RNA after infection were discussed by P. D. Cooper. Mutants resulting from treatment with 5-fluoro-uracil were selected for heat-dofectiveness over the small range of temperature of $37^{\circ}-39 \cdot 5^{\circ} \mathrm{C}$. Five to six genes affecting host cell metabolism have been detected, but the mechanism of their action is as yet little understood. Ho suggested that the proteins formed as gene products in the mutants differed in perhaps one amino-acid only and that this alteration allowed easy temperature deformation.

J. K. Pollak discussed his work on the origin of the endoplasmic reticulum in developing and regenersting liver. He presented evidence supporting the formetion of the reticulum from precursor granules (reticulosomes). These consist mainly of protein which eombines with phospholipid to form stable complexes resembling a membranous reticulum. D. E. Green discussed his accumulated knowledge of the methods of separating and re-forming the structural protein phospholipid complexes of mitochondria. He also discussed the evidence for the association of the integrated enzyme systems with structural components of the mitochondria. The localization of the effect of chloramphenicol on the formation, in $S$. cerevisiae, of completed mitochondria with respect to a marked decrease in the number of cristae and of certain mitochondrial enzymes was discussed by A. W. Linnane. The organism grows extremely well on the readily fermentable glucose. His results suggest specificity in the effect of chloramphenicol on mitochondrial dovelopment. The synthesis of chloroplasts with respect to regulation, the greening process and the involvement of contained DNA, RNA was discussed by R. M. Smillie. Once again chloramphenicol was reported to show specifieity in its effect on the synthesis of protein in the developing organelle.

Using mitochondrial swelling as a measure of substrate uptake, J. B. Chappell presented results which were consistent with the presence of carriers for malate and succinate dependent on phosphate and for citrate being dependent on the presence of L-malate in addition. He sees the process of uptake as being by exchange diffusion. Many in the audience came to sensible grips with mito. chondrial swelling for the first time.

T. P. Singer summarized the existing knowledge on the complexities of $\mathrm{NADH}_{2}$ dehydrogenase, with its flavin, non-haem iron and labile sulphur. Its functional position. in the electron transport chain in relation to phospholipid and the modifications of its properties by heat and organic solvents and mercurials were discussed.

L. P. Vernon and N. K. Boardman discussed chloroplasts and their functional components which are concerned in electron movement following photochemical excitation. They considered these components with respect to the individual steps and pathwrys involved and with respect to sub-units having definable activities. Knowledge of ehloroplast function now seems to be comparable in depth with that of mitochondria from animal tissues.
F. J. R. HrRn

\title{
CONTROL OF BIOLOGICAL DEVELOPMENT
}

$\mathrm{T}$ HE seventh Intornational Embryological Conference, sponsored by the Editorial Board of the Journal of Embryology and Experimental Morphology, was held in London during September 6-10. Three different forms of scientific communications were used: twelve main papers, five discussion groups and some eighty demonstrations were presented by scientists from twenty countries. The control of development formed the major topic of the meeting, since classical embryology is now being reinterpreted in terms of the mechanisms of control of protein synthosis by the genes. Development, of course, requires that a sequence of changes occurs in the types of synthesis carried out by a developing cell. Dr. S. Brenner (Cambridge) introduced the session on the genetics of embryogenesis by discussing the possible control mechanisms whereby a series of cistrons could bring about the sequential series of changes in synthesis required in differ- entiation. Dr. H. MacGregor (St. Andrews) described recent work on the lamp-brush chromosomes found in newt eggs during their formation. These chromosomes are intensely concerned with the synthesis of materials which form the egg.

Howevor, genetic eontrol systems of embryogenesis tend to fail even if the chromosome number is haploid or polyploid rather than diploid. Prof. L. Gallien (Paris) described a number of instances of polyploidy in amphibir and correlated these abnormalities and examples of aneuploidy with the type of failure of development observed.

Dr. L. Hamilton (London) spoke on her work on the genetic factors which may be involved in the "haploid syndrome' found in haploid amphibian embryos. This syndrome is characterized by oedema and a tendency to failure of development and is very variable in its expres. 
sion. The question is whether the syndrome is due to a lack of hybrid vigour, which would normally be found frequently in a diploid wild-type population, to the presence of certain lethal genes, active only in the haploid state, or to other causes. If it were due to the absence of hybrid vigour the syndrome would be expected to appear at equal intensity in all haploids. If, however, it were due to certain specific genes, all animals obtained by nuclear grafting from the same clone of nuclei would show the syndrome to the same extent. Dr. Hamilton reported that on performing this experiment haploid animals were obtained which showed some variation in the extent of the haploid syndrome. This result suggests that the genetic explanations suggested here are incorrect.

Gene action in development is, of course, expressed in the control of protein synthesis, and another session was devoted to protein synthesis in embryos. Dr. P. N. Campbell (London) introduced the next session by outlining the biochemical background and problems in the investigation of this topic in embryos. He pointed out that embryos might present a fruitful field for the investigation of control mechanisms since a number of morphogenetic interactions are known to involve substances, some of which are proteins, which alter the general types of synthesis in target cells and which may act directly on the genetic system.

Prof. J. Brachet (Brussels) described histochemical and autoradiographic work on the localization of protein synthesis and production of messenger RNA in the early stages of amphibian devolopment. He discussed the ways in which localization of certain substances in certain parts of the embryo might bring about locally different types of synthesis, and also considered the possibility that part of the protein synthesis might be under the control of extranuclear DNA. The amphibian egg is remarkable in that at the start of development there is roughly 1,000 times as much DNA outside the nucleus as within it.

Prof. A. Monroy (Palermo) reported his experiments on the initiation of protein synthesis in echinoderm embryos immediately following fertilization. There appear to be two separate waves of protein synthesis, one of which appears at gastrulation, as in many other embryos; but the other starts immediately after fertilization and appears to be connected with the activation of the egg.

Prof. Flickinger (Buffalo) described his experiments on the priming of RNA synthesis in different parts of the amphibian embryo at gastrulation. $\mathrm{He}$ found that priming required DNA or chromatin and suggested that protein from chromatin might tend to act as an inhibitor. Using this system he found that the endoderm appeared to be most active in synthesis of RNA. This result is rather surprising in view of the fact that simpler techniques had suggested that the dorsal lip cells were most active in RNA synthesis. Dr. B. Mintz (Philadelphia) chaired a discussion group on the initiation of synthesis (in particular protein synthesis) in the mammalian egg. The chief speakers were Dr. C. Austin (Tulane) and Dr. D. Szollosi (Philadelphia).

Another important topic at this meeting was the subject of developmental neurology. Prof. V. Hamburger (St. Louis) spoke on his work on the demonstration of spontaneous intrinsic rhythmic activity in the spinal cord of chick embryos. By separating parts of the spinal cord from the brain very early in development, it was nevertheless possible, later, to obtain motor activity in the isolated portions of the cord. This result suggests that these activities can surprisingly develop independently of control from higher centres of the contral nervous system. Prof. M. Singer (Cleveland, U.S.A.) reported on his work on the control of limb regeneration in amphibia and reptiles, and demonstrated that whether or not regeneration took place depended in part on a certain threshold of nerve mass in the stump being exceeded. The discussion group on developmental neurology was chaired by Dr. A. F. Hughes (Bristol). It opened with a review by Dr. Rita
Levi-Montalcini (St. Louis and Rome) on the sequence of her researches on the nerve growth factor. The two remaining main papers dealt with the problem of how the precise pattern of the wiring of the nervous system is established.

Dr. R. M. Gaze (Edinburgh) gave an account of his work on optic nerve regeneration in fish and amphibians, in which the restoration of the rotinotopic projection of fibres of the optic nerve on to the optic tectum is charted by means of systematic retinal stimulation, and electrical recording from the tectum. Among other experiments, Dr. Gaze showed that if half the eye rudiment is removed in early Xenopus tadpoles and a corresponding half eye grafted in its place, then fibres from the original half retina ultimately may project over the whole of the tectum. On the other hand, after hemisection of the optic nerve in the adult goldfish, there is no evidence that the projection of the remaining optic fibres spreads beyond its own half of the tectum.

The third main speaker was Dr. George Szekely (Pécs), who described recent experiments on limb movement in both urodeles and birds in which heterotopic transplantation of regions of the spinal cord were made at early stages of development. The final pattern of limb movement is greatly influenced by the source of the grafted cord. Thus, if lumbar cord is grafted at brachial levels in the chick, the wings of the young adult bird are unable to flutter when the animal is allowed to fall. Dr. Harkmark, Dr. Drachman and Prof. Singer also contributed to this discussion.

Other speakers in the main sessions were Dr. O. Vyasov (Moscow), who reported work on the immunology of embryos, Dr. D. Yaffe (Rehovoth, Israel), who described his experiments on the factors affecting musclo differentiation, and Dr. T. Humphreys (Cambridge, U.S.A.), who spoke on his experiments on factors controlling the specificity of sponge re-aggregation. Dr. N. Verdonk (Utrecht) and Dr. T. Dettlaff (Moscow) spoke on their work on early stages of molluscan and fish development respoctively. Prof. Pasteels (Brussels) read a paper by Prof. J. Mulnard (who was unable to attend) on the effect of ooplasmic segregation on the development of trophoblast or embryo in the mammalian embryo.

Three other discussion groups were held. Dr. Goss (Providence, U.S.A.) introduced a discussion on the control of organ size. Dr. A. Coulombre (Bethesda, U.S.A.) talked on his work on the control of lens and retina growth, in which he had showed by operative procedures that interactions took place between lens and retina to control their growth. Prof. W. Bullough (London) spoke of his work on the control of mitosis by chalones. In the second group, J. Dingle (Cambridge) introduced a discussion on the role of lysosomes in development. $\mathrm{He}$ suggested that, though the concept of the lysosome as a discrete intracellular organelle containing a number of hydrolytic enzymes has greatly stimulated biochemical work on the isolated particle, the functions of the lysosome are only slowly beginning to emerge. The participants in the discussion group on the ro'e of lysosomes in development clearly emphasized the importance of lysosomal enzymes in the dynamics of tissue synthesis and degradation during embryonic devolopment.

Dr. Scheib (Paris) presented electron micrographs demonstrating the presence of numerous autolosomes in the regressing Müllerian duct. These bodies, which are a form of lysosome concerned with digestion of other organelles, were large and complex and contained high acid phosphatase activity. Prof. Weber (Berno) and Dr. Eeckhout (Louvain) showed lysosomal particles to be present in the metamorphosing tadpole tail; continued synthesis of lysosomal hydrolases, particularly of protease, was found to be important in the process of regression. That changes in lysosomal activity or stability might affect other cellular functions, notably cell division, was suggested by Dr. A. C. Allison (Mill Hill). The final 
paper, by Dr. M. Prestige and Dr. A. Hughes (Bristol), dealt with the possibility that lysosomes may play a part in cell degeneration in the central nervous systom.

The very active discussion of the papers emphasized the present interest in the role of lysosomes in pathology as well as in normal physiology and embryology.

The third group was chaired by Prof. E. Wolff (Nogentsur-Marne); in the introduction to a discussion on interpretation of epigenetic malformations he pointed out that numerous physical or chemical factors, when applied at the same stage of development, produce the same types of malformations, such as cleft palates. On the contrary, other drugs have a specific teratogenic effect. The action of thalidomide on human foetuses has dramatically confirmed this point of view. The question arises of how these two different mechanisms can be explained.

Dr. W. Landauer (London) described his work on some drugs such as insulin. Dr. J. Ebert and Dr. M. Reporter (Baltimore) reported their work on the action of actinomycin $A$ on differentiation of muscles in vivo and in vitro. This substance spocifically inhibits the differentiation of the heart of the chick embryo in vivo and of muscle fibres in in vitro cultures. The authors extracted from the mito- chondrial fraction of tissues of the adult chick (liver and muscles) a protein that prevents the teratogenic effect of antimycin $A$. As a consequence this substance may be considered as inhibiting the formation of a protein essential in muscular differentiation.

Finally, Dr. Kirmann (Paris) showed that X-rays-a a non-specific teratogenic factor-affect general respiratory metabolisms, which result in a decrease of oxygen con. sumption of the tissues. This metabolic effect as well as the teratogenic action of $\mathrm{X}$-irradiation can be prevented by anoxia or by substances-such as cysteamine--which are able to combino with free radicals arising in the tissues as a result of the ionization of water. So we can assume, that the two different mechanisms of activity of teratogenic substances may be related to whether they affect general or specific motabolisms.

In addition some eighty demonstrations were presented by members of the conference, ranging over all branches of embryology. The conference was made possible by the generosity of the International Union of Biological Sciences, the Royal Society and by a number of industrial organizations. The eighth International Embryological Conference will be hold in Berne, Switzerland, in 1967.

\section{SEAWEED RESEARCH}

$\mathrm{T}$ HE fifth International Seaweed Symposium was held at Dalhousie University in Halifax during August 25-28. The purpose of these conferences has been to foster original research, both fundamental and applied, in the field of marine algae and to facilitate the presentation of such investigations. Previous symposia have been held at Edinburgh, Trondheim, Galway, and Biarritz, on a triennial cycle. In a programme of four invited lectures and 55 original communications, the topics were evenly divided between botanical, chemical, and applied aspects. A symposium within a symposium developed on the subject of algal polysaccharides.

In discussing the seaweed industry of the future, F. N. Woodward (Scotland) emphasized the possibilities of mass culture as a source of protein, the cultivation of areas for selected species as in Japan, the great need for mechanization in harvesting and drying, and the possibilities of further applications of the unique algal polysaecharides in agriculture, industry, and medicine. The use of mustard, Sinapis alba, as a test plant with which to assess growth response to seaweed extracts was described by Challen and Homingway (United Kingdom). Stephenson (United Kingdom) reported specific effects from the use of liquid seaweed fertilizer as contributing to enhanced resistance of some field crops to frost, to pathological fungi, as Botrytis on strawberries, and to animal parasites, such as aphids on beans and sugar beets.

In a masterly lecture on recent studies of the polysaccharides of Agarophytes, Araki (Japan) described his investigations of the chemical constitution of agarose and agaropectin. Sulphate groups as half-esters were established in agaropectin only. The remarkable finding of variable but appreciable amounts (1-20 per cont) of 6-O-methyl-1galactose in agarose was reported. Pyruvic acid was confirmed as a constituent of only two agaropectins. In agarose the molar ratio of D-galactose $+6-O$-methyl-Dgalactose to 3,6-anhydro-L-galactose was always unity.

Anderson and Rees (Scotland) have found that carrabiose units account for 88-99 per cent of the molecule of $x$ - and $\lambda$-carrageenens based on the products after methanolysis of native and alkali-treated matorial. A new, widoly distributed, sulphated heteroglycan, isolated first from Ascophyllum, has been discoverod by Larsen and Haug (Norway) and named ascophyllan. It contains fucoso, xylose and a uronie acid. The uneven distribution of mannuronic and guluronic acids in the main chain of alginic acid was postulated by Haug and Larsen (Norway) from investigations of partial hydrolysis with oxalic acid. The isolation of specific $x$ - and $\lambda$-carrageenases from Pseudomonas carrageenovora was reported by Yaphe et al. (Canada).

The finding of pure poly- $N$-acetylglucosamine as the only constituent of the extracellular fibres of the diatom, Thalassiosira fluviatilis, by McLachlan, MeInnes, Falk and Craigie (Canada) provides the only instance in Nature of the occurrence of this substance in pure form. It has been renamed chitan. Two other unusual compounds were reported as occurring in marine algae--dimethyl- $\beta$-propiothetin and 2,3-dibromobenzyl alcohol-4,5-disulphate.

Black et al. (Scotland) described a survey of many of the Rhodophyceae as possible sources of the carrageenans. Marked variations in yield, ratio, optical rotation, and chemical composition wore recorded. Sharp fractionation into $x$ - and $\lambda$-components with potassium chloride was not always achieved, for oxample, in Eucheuma spinosum and Polyides rotundus. Fleming, Hirst and Mannors (Scotland) have found notable similarity in the soluble and insoluble forms of laminarin except that the former exhibits a greater degree of branching. Painter (United Kingdom) presented evidence that the sulphate group in the galactose of $x$-carrageenan and furcellaran is more or less randomly distributed over all available hydroxyl groups in the carbohydrate chains and not only attacherl at C-4 as previously believed.

The unexpected property of some seaweeds to "fix" radioactive ions was described by Czapke (Poland) and Skoryna et al. (Canada). The present explanation is based on ion-exchange with soluble or insoluble alginate. This property was applied as an index of the radioactivity of the aqueous environment and as a means of inhibiting absorption of strontium-89 in the gastro-intestinal tract of animals.

In a woll-illustrated study of the lissues of Fucus, McCully (United States) pointed out differences botween the walls of the outer epidermal cells with their amorphous outor layer, the underlying parenchyma and the central medulla with its reticulum of branched primary filaments and secondary intrusive fibres, all these being embedded in a mucilaginous matrix. Observations with the light microscope, using specific stains and extended by oloctron 